\title{
Diffuse axonal injury: diffusion tensor imaging and cognitive outcome
}

\author{
Lesão axonal difusa: imagem de tensor de difusão e resultado cognitivo \\ João Gustavo Rocha Peixoto dos Santos ${ }^{1}$, João Paulo Souza de Castoํ․, Manoel Jacobsen Teixeira ${ }^{1}$, Almir \\ Ferreira de Andrade 1 , Wellingson Silva Paiva ${ }^{1}$
}

\section{Dear Editor,}

The article published by Grassi et al. ${ }^{1}$ in Arquivos de NeuroPsiquiatria has aroused great interest by presenting relevant data with a simple methodological design. The authors didactically exposed the most recent neuroimaging features of diffuse axonal injury in magnetic resonance imaging, which include diffusion tensor imaging and its most commonlyapplied analytical methods (region of interest, tractography and voxelwise analyses).

Diffuse axonal injury is one of the most recently-studied areas of traumatic brain injury research because of its impact on public health policies. Diffuse axonal injury severity is usually rated as mild, moderate or severe. Its severity presents itself as a direct risk factor for mortality and dependence of traumatic brain injury patients. Diffuse axonal injury is also believed to be the pathophysiological feature of mild traumatic brain injury persistent symptoms. Most of the symptoms of patients with mild traumatic brain injury are transient and resolve spontaneously. Nevertheless, about $30 \%$ of these patients present with psychological, cognitive or clinical symptoms three months post-injury². Conventional neuroimaging techniques are not sensitive enough to detect diffuse axonal injury, the major brain injury observed in mild traumatic brain injury. Diffusion tensor imaging is a neuroimaging technique capable of revealing microstructural axonal injuries because it is sufficiently sensitive to detect subtle changes in white matter fiber tracts. Thus, the recently-available tools are enough to diagnose diffuse axonal injury, but there is still data lacking about the cognitive prognosis of these neuroimaging findings ${ }^{3}$.

The study by Grassi et al. ${ }^{1}$ stands out for reviewing the main diffusion tensor imaging analytical techniques for the evaluation of diffuse axonal injury after traumatic brain injury. The way in which the authors present relevant information about these different methods, as well as the way important publications are discussed in each section, should be praised. The ability to choose clinical studies of diffusion tensor imaging analytical methods (region of interest, tractography, voxelwise analyses) and the exposition of their clinical results helps readers understand the current contribution of these imaging approaches in diffuse axonal injury pathophysiology, evaluation, and outcome.

The authors perfectly summed up, in the introduction of the article, when they contextualized the diffuse axonal injury impact on morbidity, presented traumatic brain injury's most common score evaluations (CRASH and International Mission for Prognosis and Analysis of Clinical Trials [IMPACT] score), and exposed diffuse axonal injury pathophysiology, remembering that it is more than an inertial mechanism of rotational acceleration-deacceleration.

The authors could have discussed more about the relationship between diffusion tensor imaging alteration and outcome. Hellstrøm et al. ${ }^{4}$ published a voxelwise analysis, showing a strong association between diffusion tensor imaging and the composite measure of self-reported score outcomes (cognitive, somatic and emotional symptoms) 12 months after mild traumatic brain injury. Galanaud et al. ${ }^{5}$ created a diffusion tensor imaging score based on fractional anisotropy, mean diffusivity, axial diffusivity, and radial diffusivity. In severe traumatic brain injury patients, this score showed a $64 \%$ sensibility and $95 \%$ specificity for the prediction of an unfavorable outcome, based on the Glasgow Outcome Scale, when compared with the IMPACT score. Net reclassification improvement and integrated discrimination improvement also demonstrated a relevant improvement in the diffusion tensor imaging score compared with the IMPACT score $(\mathrm{P}<0.001)$.

Finally, the authors' publication presented us with a distinguished article about a recently-studied point in traumatic brain injury research and they have opened the field for further longitudinal studies. That is why the article's merit must be emphasized. Some small details could be clearer in the text, but these questions do not diminish the importance of this publication for the scientific community.

${ }^{1}$ Universidade de São Paulo, Faculdade de Medicina, Departamento de Neurocirurgia, São Paulo SP, Brasil.

Correspondence: João Gustavo Rocha Peixoto dos Santos; Departamento de Neurocirurgia do HC-FMUSP; Rua Dr Eneas de Carvalho Aguiar, 255; 05407-010 São Paulo SP, Brasil; E-mail: joaogustavo@usp.br 


\section{References}

1. Grassi DC, Conceição DM, Leite CD, Andrade CS. Current contribution of diffusion tensor imaging in the evaluation of diffuse axonal injury. Arq Neuropsiquiatr. 2018 Mar;76(3):189-99. https://doi.org/10.1590/0004-282×20180007

2. Alexander MP. Mild TBI: pathophysiology, natural history, and clinical management. Neurology. 1995;45(7):253-60.

3. Benson RR, Meda SA, Vasudevan S, Kou Z, Govindarajan KA, Hanks RA, et al. Global white matter analysis of diffusion tensor images is predictive of injury severity in traumatic brain injury. J Neurotrauma. 2007 Mar;24(3):446-59. https://doi.org/10.1089/neu.2006.0153
4. Hellstrøm T, Westlye LT, Kaufmann T, Trung Doan N, Søberg HL, Sigurdardottir S, et al. White matter microstructure is associated with functional, cognitive and emotional symptoms 12 months after mild traumatic brain injury. Sci Rep. 2017 Oct;7(1):13795. https://doi.org/10.1038/s41598-017-13628-1

5. Galanaud D, Perlbarg V, Gupta R, Stevens RD, Sanchez $P$, Tollard E et al. Assessment of white matter injury and outcome in severe brain trauma: a prospective multicenter cohort. Anesthesiology. 2012 Dec;117(6):1300-10. https://doi.org/10.1097/ALN.0b013e3182755558 